\title{
Associating Liver Partition and Portal Vein Occlusion, Including Venous Congestion, Induction in Rats
}

\author{
DAISUKE KAWAGUCHI, YUKIHIKO HIROSHIMA, YUTARO KIKUCHI, \\ KENICHI MATSUO and KUNIYA TANAKA \\ Department of Surgery, Teikyo University Chiba Medical Center, Chiba, Japan
}

\begin{abstract}
Background/Aim: Associating liver partition with portal vein occlusion for staged hepatectomy (ALPPS) is a recently developed strategy for inducing rapid hypertrophy of the future liver remnant (FLR). To explore possible mechanisms, we designed the first model of ALPPS with venous congestion $(A L P P S+C)$ in rats. Materials and Methods: Rats were assigned randomly to 3 experimental groups: ALPPS, ALPPS $+C$ and sham. Hepatic regeneration rate, Ki-67 and histopathology were assessed at $24 \mathrm{~h}$, $48 \mathrm{~h}$, and 7 days postoperatively. Results: Hepatic regeneration rate was much higher for ALPPS $+C$ than for ALPPS at $48 \mathrm{~h}$ and 7 days postoperatively $(p<0.01)$. Microscopically, the regenerating liver showed greater hepatocyte density and smaller hepatocyte size in ALPPS $+C$ than in ALPPS $(p<0.01$ for each). Conclusion: Greater hepatic regeneration in $A L P P S+C$ than in ALPPS confirmed that we established a rat model of ALPPS with benefit from venous congestion. Producing a congested area may contribute importantly to rapid FLR hypertrophy during ALPPS.
\end{abstract}

Liver resection remains the only potentially curative option for patients with primary or secondary liver tumors. Surgeons continue to expand the limits of "resectable" disease by performing extensive liver resections that challenge both the oncologic and technical definitions of resectability. Within this context of extended resection, postoperative liver failure arising from insufficient function of the future liver remnant (FLR) remains a major concern. Multiple strategies for increasing FLR size to reduce risk of postoperative liver failure have been applied.

Correspondence to: Kuniya Tanaka, MD, Department of Surgery, Teikyo University Chiba Medical Center, 3426-3 Anesaki, Ichihara, Chiba, 299-0111, Japan. Tel: +81 436621211, Fax: +81 436613961, e-mail: U17-92TS@med.teikyo-u.ac.jp

Key Words: ALPPS, congestion, liver regeneration, hepatectomy, portal vein occlusion, rats.
Associating liver partition and portal vein occlusion for staged hepatectomy (ALPPS) was recently found to enhance volume increase in the FLR more rapidly than that obtained with previous techniques, such as portal vein embolization (PVE) followed by hepatectomy or classical 2-stage hepatectomy (1).

Until now, exact mechanisms underlying the remarkable regenerative response of the FLR in ALPPS are not disclosed. After the first resection of ALPPS, enhancement of the effect of portal vein (PV) occlusion by interruption of vascular intercommunications during parenchymal transection, rapid atrophy of the completely avascular area after division of portal pedicles during transection as in segment (S) 4 during right (rt.) liver plus $\mathrm{S} 4$ resection, as well as producing a significant area with inflammatory injury, are thought to contribute to intense stimulation of liver regeneration $(2,3)$. However, a completely avascular area could sometimes become a focus of life-threatening sepsis. Another possible mechanism involves the area of congestion induced by interruption of hepatic vein branches during parenchymal partition. The area of venous congestion may develop considerable atrophy comparable to that in the completely ischemic area, resulting in compensatory hypertrophy of the FLR. We speculate that this congested area is possibly critical to FLR hypertrophy in ALPPS. However, little information is available regarding how closely the congested area is related to the increase in FLR volume.

In the present study, we established a model of ALPPS with venous congestion in rats to explore possible mechanisms of the spectacular regenerative response in ALPPS.

\section{Materials and Methods}

Animals. Eight-week-old male Wistar rats (Kureo, Tokyo, Japan) were maintained in a room with a 12-h light/dark cycle and given free access to tap water and laboratory food. During all experimental procedures, the animals were treated in accordance with the guidelines outlined in the Guide for the Care and Use of Laboratory Animals prepared by the National Academy of Sciences and published by the National Institutes of Health (Bethesda, MD, USA). 
The experimental protocol has been reviewed and approved by our institutional animal care committee (Protocol Number: 15-025).

Experimental model of operative procedure. Rat livers have 4 lobes: rt. lobe (RL), middle lobe (right middle lobe (RML); and left middle lobe (LML)), left lateral lobe (LLL) and caudate lobe ((CL); Figure 1a). Each lobe is irrigated by its own portal pedicle and drained by its own hepatic veins. In turn, the middle lobe has 2 separate PV branches: a middle lobe right branch and a middle lobe left branch. Based on this anatomy, we developed an ALPPS experimental model that focused on the middle lobe in conducting ALPPS, since the shape and anatomy of the middle lobe are similar to those in human liver (4). All animals were anaesthetized with isoflurane and oxygen (concentration of isoflurane $1.5 \%$ at a flow rate of $0.51 / \mathrm{min}$ ). Rats were assigned randomly to 1 of 3 experimental groups: sham operation (SHAM), ALPPS and ALPPS with venous congestion (ALPPS+C).

In SHAM animals, the hepatic artery, PV and bile duct were dissected without ligation, after which the abdomen was closed by a double running suture (Figure 1a). In ALPPS animals, selective PVL was performed on the CL, LLL and LML, as well as RL. After careful dissection of the hepatic artery and bile duct, corresponding portal veins of the lobes were suture-ligated with 6-0 silk. The RML was preserved to regenerate. When the common trunk of the PV of the LLL and LML was ligated, the ischemic line (i.e., the border) emerged immediately to the right of the falciform ligament. Hepatic parenchymal transection then was performed along this ischemic demarcation line using bipolar scissors; these steps were performed successively until the paracaval lobe was reached (Figure 1b) (5). In ALPPS+C animals, PVL and hepatic parenchymal transection were performed as in ALPPS animals. Then, venous congestion was induced by ligation of the left hepatic vein (Figure 1c).

Arterial circulation and biliary duct branches were maintained in all animals. No deaths or serious complications occurred during operation or the subsequent observation period (7 days).

Experimental design and operative procedures. Animals were sacrificed at $24 \mathrm{~h}, 48 \mathrm{~h}$ and 7 days after the operation (6 rats from each group per time point). At that time, liver samples were obtained for histologic assessment. The entire liver was removed and divided into the RML, LML and the other lobes (LLL, RL and CL; OL). After weighing, approximately $200 \mathrm{mg}$ of liver tissues from the RML and LML were fixed in $10 \%$ formaldehyde or snapfrozen in liquid nitrogen, with the frozen samples being stored at $-80^{\circ} \mathrm{C}$ until use.

Measurements. To determine the effects of ALPPS and ALPPS $+\mathrm{C}$ on liver regeneration, we weighed the RML (WR), LML (WL) and OL (WO) using a laboratory micro-scale at each time point. Weights before surgery (preWR, preWL, and preWO) and those after surgery (sham operation or liver transection and PV ligation with or without vein ligation; postWR, postWL, and postWO) were obtained. The percentage increase or decrease in volume was calculated with the following formulas: RML $\%=[($ postWR - preWR $) /$ preWR $] \times 100$, $\mathrm{LML} \%=[($ postWL - preWL $) /$ preWL $] \times 100$ or $\mathrm{OL} \%=[($ postWO preWO)/preWO] $\times 100$.

Histologic examination. Liver tissues were immersion-fixed in $10 \%$ formaldehyde, embedded, sectioned and stained with hematoxylineosin (H-E). In the RML and LML at $48 \mathrm{~h}$ after operation, sinusoidal narrowing or dilation, hepatocyte density and size of hepatocytes were assessed. With sinusoidal narrowing or dilation in the SHAM model used to define "none", sinusoidal changes after each procedure were assigned a grade (none, slight, moderate or severe) according to severity of findings (6). The severe grade was defined as positive. Hepatocyte density was determined by counting hepatocytes in 4 high-power fields ( $x 400$, averaged). Size of hepatocytes was determined by measuring 10 hepatocytes in highpower fields (x400, averaged). For these analyses of density and size, non-commercial image processing software, (ImageJ; http://imagej.nih.gov/ij/) (7), was used.

Liver sections from the RML taken at $24 \mathrm{~h}, 48 \mathrm{~h}$ and 7 days after operation were deparaffinized and rehydrated with xylene and a graded ethanol series. Antigen retrieval was performed in $10 \mathrm{mM}$ sodium citrate buffer ( $\mathrm{pH} \mathrm{6.0)}$ in a microwave oven at $121^{\circ} \mathrm{C}$ for 30 minutes. Endogenous peroxidase activity was quenched by immersion in $0.3 \%$ hydrogen peroxide in methanol for 30 minutes. After non-specific binding sites were blocked with $10 \%$ rabbit serum solution for 15 minutes at $37^{\circ} \mathrm{C}$, sections were incubated at room temperature for $1 \mathrm{~h}$ with a mouse monoclonal anti-rat Ki-67 antibody diluted 1:25 in phosphate-buffered saline (DAKO Japan Co. Ltd., Tokyo, Japan). The sections were then incubated with EnVision anti-mouse (Dakocytomation EnVision1System labeled polymer-HRP anti-mouse), followed by incubation with 3,3'diaminobenzidine and counterstained with Mayer's hematoxylin. $\mathrm{Ki}-67$-positive hepatocytes were counted in 10 randomly chosen high-power fields and the counts were averaged. All histologic analyses were performed in a blinded fashion with respect to experimental group.

Real-time reverse-transcription polymerase chain reaction (RT$P C R)$. To determine the effects of ALPPS and ALPPS $+\mathrm{C}$ on liver regeneration, we measured cytokines associated with hepatocyte proliferation in the RML. RT-PCR was performed at $48 \mathrm{~h}$ after operation in RML tissue to quantify messenger RNA (mRNA) expression for the genes encoding: tumor necrosis factor- $\alpha(\operatorname{Tnf}-\alpha)$ and interleukin-6 (Il-O). Total RNA was isolated from each sample using an RNAspin mini RNA isolation kit (GE Healthcare, Buckinghamshire, UK) and cDNA was synthesized from $2 \mathrm{mg}$ of each total RNA sample using High Capacity RNA-to-DNA kits (Applied Biosystems, Foster City, CA, USA). Each cDNA sample was diluted 4-fold before PCR amplification. Primers were designed using TaqMan Gene Expression Assays (Applied Biosystems). RTPCR was performed using a 7900HT Fast RT-PCR System (Applied Biosystems) and TaqMan Fast Advanced Master Mix (Applied Biosystems) according to the manufacturer's instructions. The amplification protocol consisted of an initial 10-min denaturation at $95^{\circ} \mathrm{C}$, followed by 40 cycles of denaturation at $95^{\circ} \mathrm{C}$ for $10 \mathrm{sec}$, annealing at $60^{\circ} \mathrm{C}$ for $40 \mathrm{sec}$ and extension at $72^{\circ} \mathrm{C}$ for $10 \mathrm{sec}$. Degree of expression for each target gene was normalized relative to that for the glyceraldehyde-3-phosphate dehydrogenase (GAPDH) gene in the same sample. Assays were performed in triplicate. Results are presented as an $\mathrm{x}$-fold induction relative to baseline expression in the SHAM group.

Statistical analysis. Continuous data are expressed as mean $( \pm \mathrm{SD})$ or median (range), and were analyzed using the Mann-Whitney $U$-test. The $\chi^{2}$ test or Fisher's exact test was used for analysis of categorical variables. A difference was considered significant when the 2 -sided $p$-value was below 0.05 . 
a

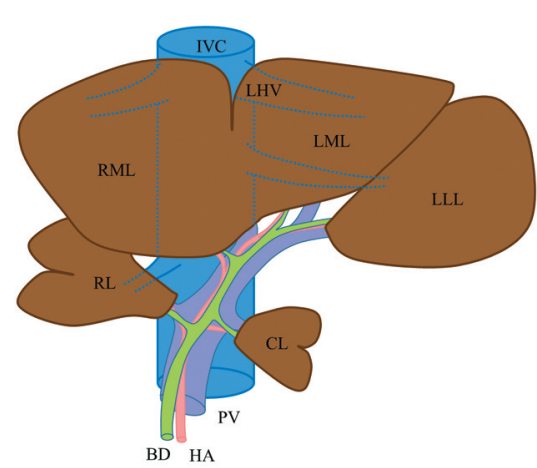

b

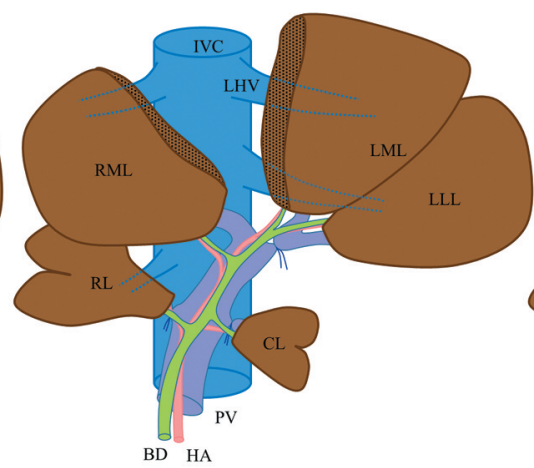

C

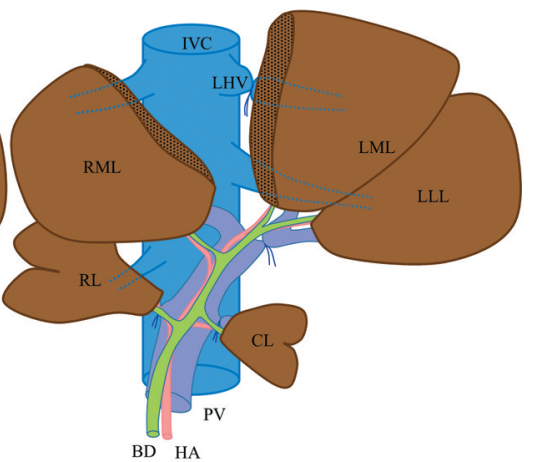

Figure 1. Surgical procedure for ALPPS and for ALPPS with venous congestion models in the rat. Preoperative rat liver anatomy is shown in panel a. In ALPPS (b), PVL is performed at the caudate lobe, left middle and left lateral lobe, as well as right lobe. Note the demarcation between the normally perfused right median lobe and the portal-depleted left median lobe, where transection is performed. In ALPPS with venous congestion (c), the same procedure (PVL and hepatic parenchymal transection) carried out in ALPPS animals is performed, followed by venous congestion induction by ligation of the left hepatic vein. ALPPS, associating liver partition and portal vein occlusion for staged hepatectomy, CL indicates caudate lobe; RML, right middle lobe; LML, left middle lobe; LLL, left lateral lobe; PV, portal vein; IVC, inferior vena cava; HA, hepatic artery; LHV, left hepatic vein.
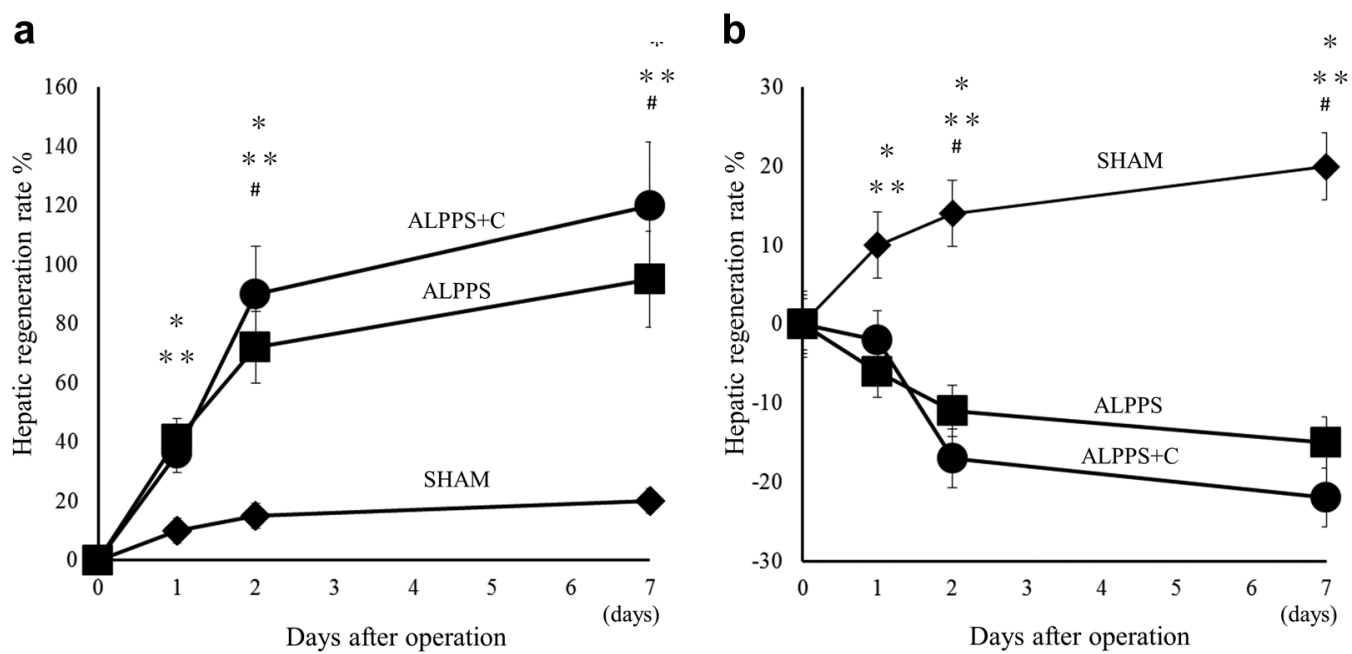

Figure 2. Hepatic regeneration rate of the RML and LML after SHAM, ALPPS and ALPPS+C. The hepatic regeneration rate in ALLPS and $A L P P S+C$ was significantly greater than that of the SHAM group at 1,2, and 7 days, while the hepatic regeneration rate of ALPPS $+C$ was significantly greater than that of the ALPPS group at 2 and 7 days. Values are means $\pm S D$. " $p<0.05$ for the ALPPS $+C$ group vs. the ALPPS group; ${ }^{*} p<0.05$ for the ALPPS $+C$ group vs. the SHAM group; ** $<<0.05$ for the ALPPS group vs. the SHAM group. ALPPS, associating liver partition and portal vein occlusion for staged hepatectomy; ALPPS $+C$, ALPPS with venous congestion; RML, right middle lobe; LML, left middle lobe.

\section{Results}

Change in liver volume. PreWR, preWL and preWO for each rat were calculated as the animal's weight $\times 0.69,0.29$ or $1.11 \%$ (these 3 percentages, respectively, were the mean percentage of the body weight represented by the RML, LML and OL in 10 normal male Wistar rats weighing 250 to $300 \mathrm{~g}$ ).
RML weights for both ALPPS and ALPPS+C were clearly higher than for SHAM at all time points. There was no difference in RML volume percentage between the ALPPS group $(41.1 \pm 8.8 \%)$ and the ALPPS+C group $(37.4 \pm 7.1 \%)$ at $24 \mathrm{~h}$ after operation. However, compared to the ALLPS group, ALPPS $+\mathrm{C}$ induced a greater regeneration response with an increased RML volume percentage at 2 days after 


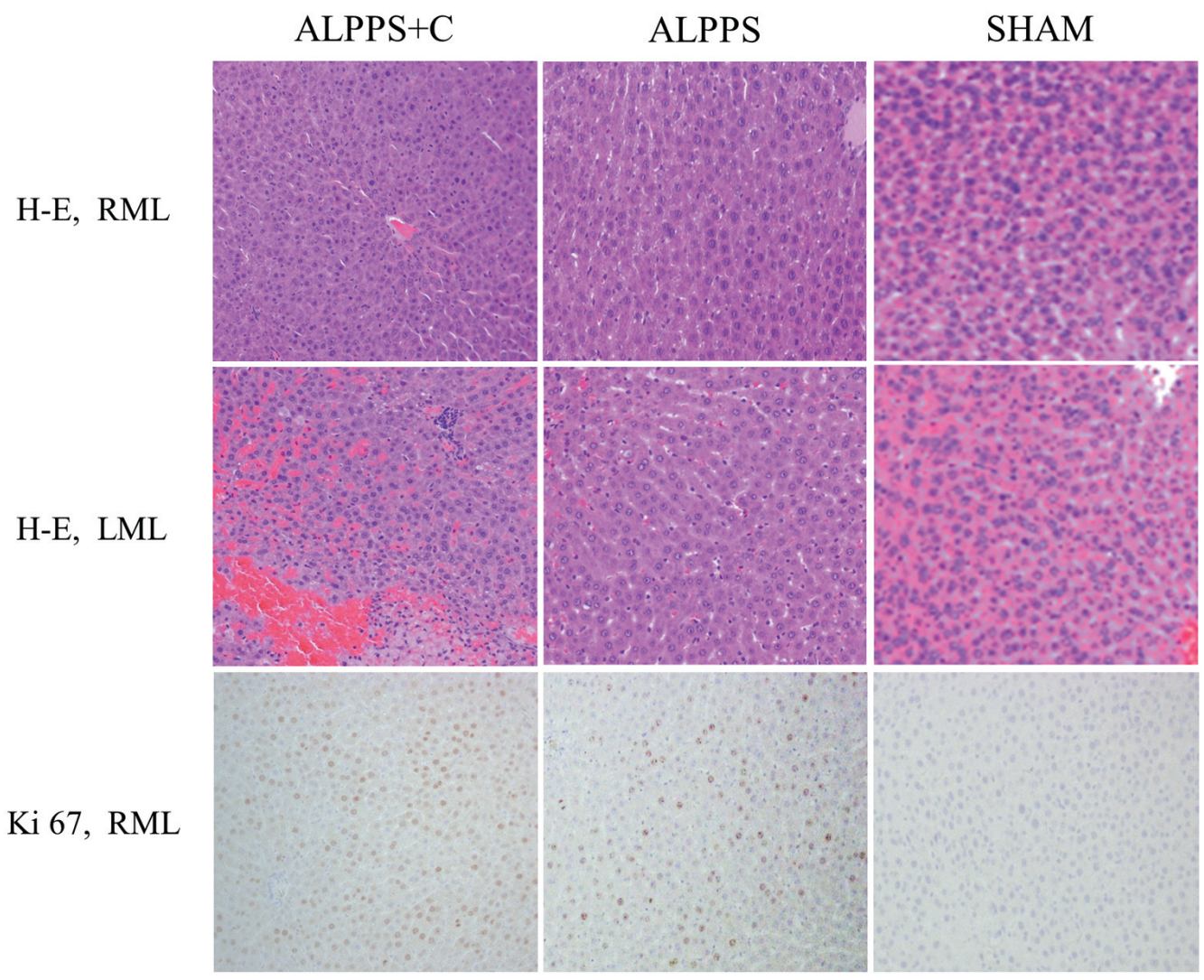

Figure 3. Hematoxylin and eosin (H-E) staining. H-E staining in the RML and LML, as well as Ki-67-positive hepatocytes in the RML, on day 2 after the procedures $(\times 100)$. ALPPS, Associating liver partition and portal vein occlusion for staged hepatectomy; ALPPS $+C, A L P P S$ with venous congestion; RML, right middle lobe; LML, left middle lobe.

operation $(90.5 \pm 15.7 \%$ vs. $72.4 \pm 11.1 \%, p<0.01)$ and at 7 days after operation $(120.2 \pm 22.7 \%$ vs. $95.5 \pm 17.9 \%, p<0.01)$ (Figure 2a). Compared to the ALLPS group, ALPPS $+\mathrm{C}$ reduced LML volume percentage at 2 days after operation $(-11.1 \pm 2.6 \%$ vs. $-17.5 \pm 3.9 \%, p<0.05)$ and at 7 days after operation $(-22.8 \pm 5.7 \%$ vs. $-15.1 \pm 3.2 \%, p<0.05)$, although no difference in LML volume percentage between the ALPPS group and the ALPPS $+C$ group was observed at $24 \mathrm{~h}$ after operation $(-6.2 \pm 2.7 \%$ vs. $-2.4 \pm 1.1 \%, p=0.548$; Figure $2 b$ ). Meanwhile, no significant difference in the OL volume percentage was evident between the 2 groups at all time points $(-14.8 \pm 3.1 \%$ vs. $-13.8 \pm 2.9 \%, p=0.687$ at 24 hour, $-34.5 \pm 6.9 \%$ vs. $-35.4 \pm 7.3 \%, p=0.612$ at 2 days and $-40.8 \pm 8.8 \%$ vs. $-39.9 \pm 8.4 \%, p=0.551$ at 7 days $)$.

Histologic assessment. Representative photomicrographs at 2 days (H-E and Ki-67) are shown in Figure 3. Sinusoidal narrowing in the RML was observed more frequently in ALPPS+C (75\%) and ALPPS (50\%) than in SHAM (0\%; $p=0.014$ and 0.046 , respectively). Sinusoidal dilation in the

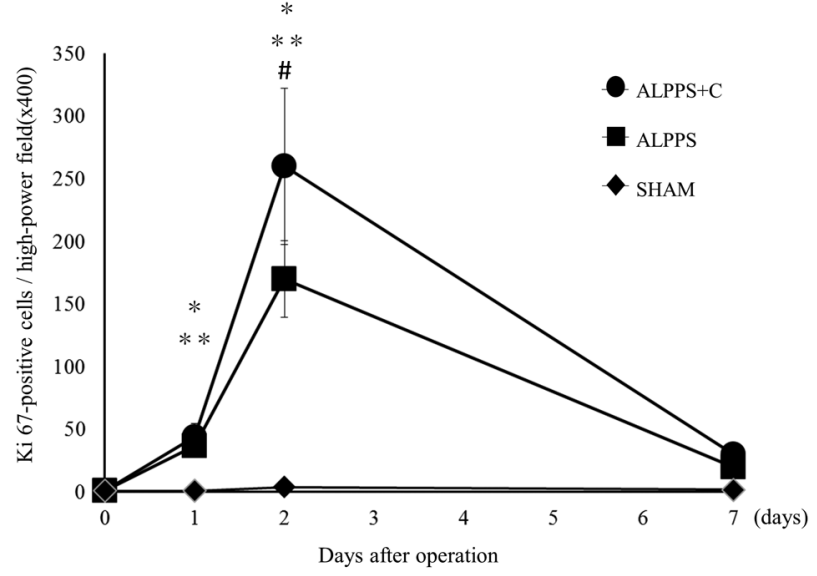

Figure 4. Numbers of Ki-67-positive hepatocytes in RML at all time points. The number of positive hepatocytes per high-power field is presented. " $p<0.05$ for the ALPPS $+C$ group vs. the ALPPS group; $* p<0.05$ for the ALPPS $+C$ group vs. the SHAM group; ** $<<0.05$ for the ALPPS group vs. the SHAM group. ALPPS, associating liver partition and portal vein occlusion for staged hepatectomy; $A L P P S+C$, ALPPS with venous congestion; RML, right middle lobe. 
Kawaguchi et al: Venous Congestion and ALPPS in Rats

Table I. Pathologic findings in paraffin-embedded specimens from RML and LML stained with hematoxylin and eosin at 2 days after operation

\begin{tabular}{|c|c|c|c|}
\hline Findings & $\begin{array}{c}\text { ALPPS+C } \\
(\mathrm{n}=6)\end{array}$ & $\begin{array}{l}\text { ALPPS } \\
(\mathrm{n}=6)\end{array}$ & $\begin{array}{c}\text { SHAM } \\
(\mathrm{n}=6)\end{array}$ \\
\hline \multicolumn{4}{|l|}{ RML } \\
\hline Narrowing of sinusoids & $4(75 \%)^{*}$ & $3(50 \%) * *$ & 0 \\
\hline Hepatocyte density, cells/HPF & $\begin{array}{c}236.7 \pm 51.2 * \# \\
(223,214-355)\end{array}$ & $\begin{array}{c}176.7 \pm 36.2 * * \\
(167,139-234)\end{array}$ & $\begin{array}{c}122.6 \pm 19.4 \\
(117,94-144)\end{array}$ \\
\hline Size of hepatocytes, $\mu \mathrm{m}$ & $\begin{array}{c}16.7 \pm 2.2 * \# \\
(15.1,11.2-20.5)\end{array}$ & $\begin{array}{c}21.7 \pm 4.5^{* *} \\
(21.5,19.3-27.8)\end{array}$ & $\begin{array}{c}30.9 \pm 7.2 \\
(30.1,27.3-35.8)\end{array}$ \\
\hline \multicolumn{4}{|l|}{ LML } \\
\hline Sinusoidal dilation & $6(100 \%)^{* \#}$ & $1(16 \%)$ & 0 \\
\hline Hepatocyte density, cells/HPF & $\begin{array}{c}80.7 \pm 12.2 * \# \\
(78,74-99)\end{array}$ & $\begin{array}{c}125.1 \pm 20.9 \\
(119,104-144)\end{array}$ & $\begin{array}{c}119.6 \pm 18.7 \\
(107,99-131)\end{array}$ \\
\hline Size of hepatocytes, $\mu \mathrm{m}$ & $\begin{array}{c}24.3 \pm 5.1^{*} \\
(24.1,22.2-29.5)\end{array}$ & $\begin{array}{c}29.1 \pm 6.2 \\
(28.6,25.3-35.5)\end{array}$ & $\begin{array}{c}31.5 \pm 7.4 \\
(30.8,28.3-36.8)\end{array}$ \\
\hline
\end{tabular}

Values of continuous variables are means \pm SD. Medians and ranges are shown in parentheses. Hepatocyte density was determined by counting hepatocytes in 4 high-power fields ( $\times 400$, averaged). Size of hepatocyte was determined by measuring 10 hepatocytes in high-power fields $(x 400$, averaged). ${ }^{*} p<0.01$, ALPPS $+C$ group $v s$. ALPPS group; ${ }^{*} p<0.01$, ALPPS $+C$ group $v s$. SHAM group; ${ }^{*} p<0.01$, ALPPS group $v s$. SHAM group. ALPPS, associated liver partition and portal vein ligation for staged hepatectomy; ALPPS+C, ALPPS with venous congestion; RML, right median lobe; LML left median lobe; HPF, high-power field.

LML was observed more frequently in ALPPS+C (100\%) than in SHAM $(0 \% ; p<0.01)$. Hepatocyte density in the RML was greater and hepatocyte size was smaller in the ALPPS $+C$ group than in the ALPPS group $(p<0.01$ for each). Hepatocyte density in the LML was smaller in the ALPPS $+C$ group than in the ALPPS group ( $p<0.01$, Table I). The number of Ki-67-positive hepatocytes per high-power field in the regenerating liver lobe (RML) was greater in the ALPPS $+C$ group than in the ALPPS group at 48 hours after operation $(260.3 \pm 57.5 v s$. 171.1 $\pm 36.6, p=0.048)$ but no significant change was observed at $24 \mathrm{~h}(44.2 \pm 9.5 \mathrm{vs}$. $37.6 \pm 7.2, p=0.452)$ or at 7 days after operation $(30.5 \pm 5.9 v s$. $20.1 \pm 3.4, p=0.122$; Figure 4).

Up-regulation of $m R N A$ expression for pro-inflammatory cytokines. Il- 6 and Tnf- $\alpha$ mRNA were highly up-regulated in the regenerating lobes $48 \mathrm{~h}$ after ALPPS group or ALPPS+C group compared to SHAM. In addition, Tnf- $\alpha$ mRNA expression was significantly greater in the ALPPS $+C$ group than in the ALPPS group $(4.1 \pm 1.2$ vs. 1.6 $\pm 0.6, p<0.01$; Figure 5).

\section{Discussion}

Volume and kinetic growth of the FLR have been reported to increase more dramatically in ALPPS than in classical 2stage hepatectomy $(1,2,8,9)$. Complete devascularization of an area, such as S4 in rt. liver plus S4 resection, represents a strong stimulus leading to rapid and marked hypertrophy of the FLR during ALPPS (10). However, such a devascularized ischemic area can become a nidus for lifethreatening sepsis (10-13). Therefore, although ALPPS fosters a rapid regenerative response, it raises concern about increased morbidity and mortality $(3,8,11)$. To avoid creating such an ischemic area, by complete devascularization, partial transection of parenchyma avoiding dissection of thick portal pedicles (partial ALPPS) (14) or preservation of major portal pedicles that emerge at the plane during parenchymal transection (15) should be considered. However, extent of FLR hypertrophy representing liver regeneration might be smaller when devascularized complete ischemia is avoided.

An alternative stimulus to regeneration involves the area of congestion induced by interruption of hepatic vein branches during parenchymal partition. This area of venous congestion experiences considerable atrophy, resulting in compensatory hypertrophy of the FLR. We believe that producing this congested area is a way to strongly stimulate FLR hypertrophy in ALPPS. In this study, we reproduced some of the physiologic consequences of ALPPS with venous congestion in a rat model. Although tumor clearing in FLR was not added in the model, the model was assumed to ALPPS procedure consisted with parenchymal transection in a plane of main portal fissure and ligation of hepatic vein (i.e. middle hepatic vein) within the deportalized liver with several tumor clearings in the FLR.

A greater regeneration response with an increased FLR (RML) volume percentage at 2 and 7 days after surgery was observed in the ALPPS $+\mathrm{C}$ group than the ALPPS group. This increase was directly related to a reduction in volume 
a

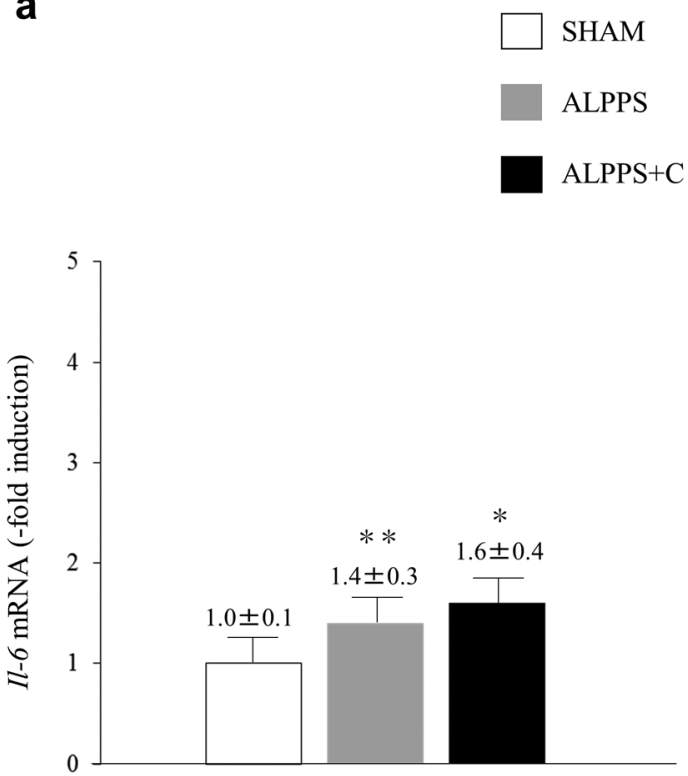

b

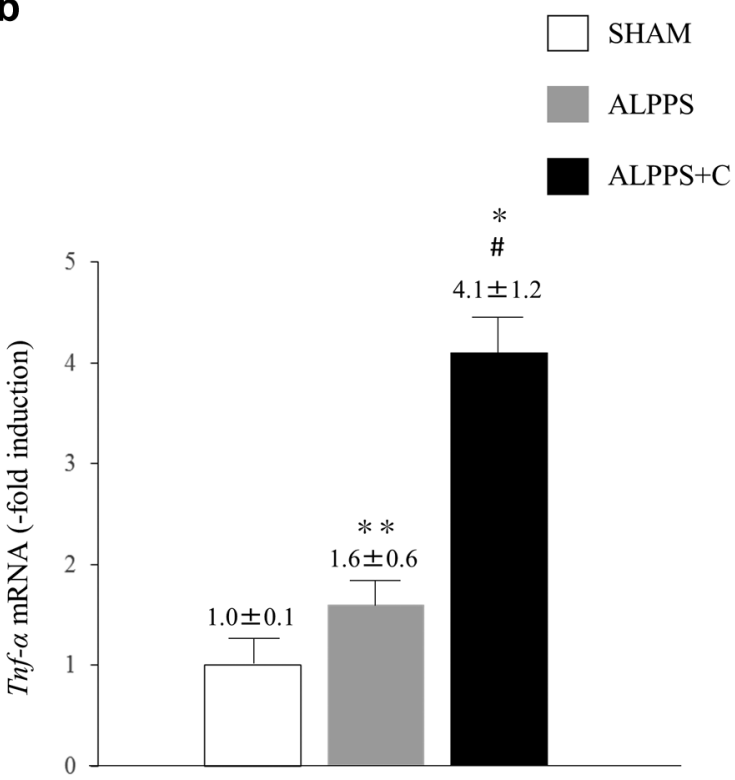

Figure 5. Expression of mRNA for cytokines in RML tissue at 2 days after the procedures. Relative mRNA expression of Il- 6 and Tnf- $\alpha$, determined by RT-PCR, is given as multiples of expression in sham-operation livers. ${ }^{*} p<0.05$ for the ALPPS $+C$ group vs. the ALPPS group; * $p<0.05$ for the $A L P P S+C$ group $v s$. the SHAM group; **p<0.05 for the ALPPS group vs. the SHAM group. ALPPS, associating liver partition and portal vein occlusion for staged hepatectomy; ALPPS $+C$, ALPPS with venous congestion; RML, right middle lobe; LML, left middle lobe; RT-PCR, real-time reverse-transcription polymerase chain reaction.

of the liver portion that underwent venous congestion (LML). PVL blocks portal flow to the congested lobe, with blood flow in the congested lobe being maintained only by hepatic arterial flow. Further, hepatic arterial buffering response, commonly induced by PV occlusion (16), was regulated by hepatic vein ligation in the ALPPS $+\mathrm{C}$ as a gradual decrease of arterial blood flow might contribute to produce more atrophy than the original ALPPS. Producing congestion-atrophy in the deportalized liver by interruption of draining veins can, therefore, be an effective way to induce FLR hypertrophy after the first procedure in ALPPS.

Meanwhile, in the FLR (RML), H-E-stained sections showed greater hepatocyte density and smaller hepatocyte size in the ALPPS $+\mathrm{C}$ group than in the ALPPS group. Narrowing of sinusoidal spaces by increased numbers of hepatocytes reflected cell proliferation in the ALPPS $+C$ group. A higher $\mathrm{Ki}-67$ labeling index in ALPPS+C than in ALPPS is consistent with greater hepatocyte proliferation in the ALPPS $+C$ group, representing greater compensatory hypertrophy of the FLR.

Tnf- $\alpha$ mRNA expression in the regenerative lobe (RML) showed greater up-regulation in the ALPPS+C group than in the ALPPS group. Tnf- $\alpha$ has been reported to be a likely primer of hepatocyte proliferation that increases post-resection liver regeneration (17). This suggests that venous congestion could promote the initiation of liver regeneration through up- regulating this pro-inflammatory cytokine, in addition to the interaction between atrophy in the congested zone and compensative hypertrophy in the FLR. As previously reported, liver regeneration is a process that involves multiple intracellular events that show complex interactions with cytokines and growth factors $(18,19)$. This possibility is consistent with the results of our study, which indicated that the mRNA expression for Tnf- $\alpha$ and $l l-6$ in regenerating liver tissues was higher in both the ALPPS and the ALPPS+C group than in the SHAM group. Among proposed factors influencing early future liver remnant (RML) regeneration, the present results support our hypothesis that an area of congestion in the deportalized liver provides an important regenerative stimulus during ALPPS $+\mathrm{C}$ treatment.

In conclusion, this study provides important new information concerning the significance of the congested area produced in the modified ALPPS procedure for stimulating volumetric recovery. Sepsis originating in the congested area produced by middle hepatic vein interruption in the implanted right liver graft after live donor liver transplantation has been reported (20). However, there have been no reports that the congested area within deportalized liver induces fatal sepsis. Therefore, we believe that this area of congestion is clinically important for successful ALPPS. 


\section{Financial Disclosures}

The Authors have no financial disclosures.

\section{References}

1 Schnitzbauer AA, Lang SA, Goessmann H, Nadalin S, Baumgart J, Farkas SA, Fichtner-Feigl S, Lorf T, Goralcyk A, Horbelt R, Kroemer A, Loss M, Rummele P, Scherer MN, Padberg W, Konigsrainer A, Lang H, Obed A and Schlitt HJ: Right portal vein ligation combined with in situ splitting induces rapid left lateral liver lobe hypertrophy enabling 2-staged extended right hepatic resection in small-for-size settings. Ann Surg 255: 405414, 2012.

2 de Santibanes E and Clavien PA: Playing Play-Doh to prevent postoperative liver failure: The “ALPPS"approach. Ann Surg 255: 415-417, 2012.

3 Alvarez FA, Ardiles V and de Santibanes E: The ALPPS approach for the management of colorectal carcinoma liver metastases. Curr Colorectal Cancer 9: 168-177, 2013.

4 Kogure K, Ishizaki M, Nemoto M, Kuwano H and Makuuchi M: A comparative study of the anatomy of rat and human livers. J Hepatobiliary Pancreat Surg 6: 171-175, 1999.

5 Dahmen U, Madrahimov N, Madrahimova F, Ji Y, Schenk A and Dirsch O: Small for size syndrome in the rat: Does size or technique matter? J Surg Res 149: 15-26, 2008.

6 Baak J P A and Oort J: A manual of morphometry in diagnostic pathology. Berlin, Heidelberg, New York, Tokyo: SpringerVerlag, 1983.

7 Rasband WS: ImageJ, US National institutes of health, Bethesda, Maryland, USA, 1997-2012. http://imagej.nih.gov/ij/

8 Li J, Girotti P, Königsrainer I, Ladurner R, Königsrainer A and Nadalin S: ALPPS in right trisectionectomy: A safe procedure to avoid postoperative liver failure? J Gastrointerest Surg 17: 956961, 2013.

9 Sala S, Ardiles V, Ulla M, Alvarez F, Pekolj J and de Santibañes E: Our initial experience with ALPPS technique: Encouraging results. Updates Surg 64: 167-172, 2012.

10 Ratti F, Cipriani F, Gagliano A, Catena M, Paganelli M and Aldrighetti L: Defining indications to ALPPS procedure: Technical aspects and open issues. Updates Surg 66: 41-49, 2014.
11 Andriani OC: Long-term results with associating liver partition and portal vein ligation for staged hepatectomy (ALPPS). Ann Surg 256: e5, 2012.

12 Tanaka $\mathrm{K}$ and Endo I: ALPPS: short-term outcome and functional changes in the future liver remnant. Ann Surg 262: e88-89, 2015.

13 Hernandez-Alejandro R, Bertens KA, Pineda-Solis K and Croome KP: Can we improve the morbidity and mortality associated with the associating liver partition with portal vein ligation for staged hepatectomy (ALPPS) procedure in the management of colorectal liver metastases? Surgery 157: 194201, 2015.

14 Petrowsky H, Gyori G, de Oliveira M, Lesurtel M and Clavien PA: Is partial-ALPPS safer than ALPPS? A single-center experience. Ann Surg 261: e90-92, 2015.

15 Tanaka, K, Kikuchi Y, Kawaguchi D, Murakami T, Hiroshima Y and Matsuo K: Modified ALPPS procedures avoiding division of portal pedicles. Ann Surg 265: e14-20, 2016.

16 Suzuki S, Nakamura S, Sakaguchi T, Ochiai H, Konno H, Baba $\mathrm{S}$ and Baba S: Alteration of reticuloendothelial phagocytic function and tumor necrosis factor-alpha productionafter total hepatic ischemia. Transplantation 64: 821-827, 1997.

17 Webber EM, Bruix J, Pierce RH and Fausto N: Tumor necrosis factor primes hepatocytes for DNA replication in the rat. Hepatology 28: 1226-1234, 1998.

18 Fausto N: Liver regeneration. J Hepatol 32: 19-31, 2000.

19 Michalopoulos GK: Liver regeneration. J Cell Physiol 213: 286300, 2007.

20 Lee S, Park K, Hwang S, Lee Y, Choi D, Kim K, Koh K, Han S, Choi K , Hwang K, Makuuchi M, Sugawara Y and Min P: Congestion of right liver graft in living donor liver transplantation. Transplantation 71: 812-814, 2001.

Received March 23, 2017

Revised April 25, 2017

Accepted April 26, 2017 